\title{
Europa bewegt auch die Allergologen
}

E uropa beschäftigt nicht nur die Bundesbürger und die Politiker wegen Maastricht und Euro. Europa beschäftigt auch die Standespolitiker, hier u.a. auch die allergologischen Gesellschaften. In Brüssel laufen die verschiedenen Vorschläge im Rahmen der Union Européenne de Medicin Specialistes (UEMS) zusammen. Auf der gerade zu Ende gegangenen Tagung der European Academy of Allergology and Clinical Immunology (EAACI) fanden drei Treffen zu diesem Thema statt. Wichtigstes derzeitiges Fazit:

In den Bestrebungen um einen europäischen Allergiespezialisten wird es auf der Basis eines Facharztes - in den romanischen Ländern meist Innere Medizin, in Mitteleuropa sind auch andere Disziplinen, wie Dermatologie, Pädiatrie etc. möglich - eine zweijährige Spezialisierung in Allergologie geben. In der davorliegenden, mindestens vierjährigen Facharztweiterbildung kommt einem "Common Trunc" besondere Bedeutung zu, der allergologische Inhalte im Rahmen der Weiterbildung zum Facharzt beschreibt. Die zwischenzeitlich als Modell diskutierte stark laboratoriums-immunologisch geprägte Weiterbildung mit lediglich kurzem klinischen Ende (3 Monate Dermatologie, 3 Monate Pädiatrie, 3 Monate Pneumologie etc.) wird nicht länger favorisiert. Unklar sind noch die Kriterien, die eine Institution zur Weiterbildung ermächtigen. Dennoch ist es als Fortschritt zu betrachten, daß in der europäischen Gesamtheit bestimmte national gewachsene Traditionen auch in Zukunft Berücksichtigung finden.

Insgesamt tröstet und beunruhigt die europäische Begegnung gleichermaßen. Die deutschen Allergologen stehen mit ihren großen Problemen nicht allein. Die grundsätzlichen Schwierigkeiten sind in ganz Europa ähnlich, mit unterschiedlichen Nuancen. Die schwindenden Ressourcen zwingen allenthalben $\mathrm{zu}$ drastischen Einsparungen und führen zu verschärf- ten Verteilungskämpfen. Dabei ist eines klar: Wenn eine kleine Disziplin, die gerade erst um ihre Definition ringt, sich noch in sich in Zwistigkeiten verzettelt, wird sie in der großen Auseinandersetzung wenig Chancen haben. Um so erfreulicher ist es, daß in Deutschland ein enger Schulterschluß zwischen der Deutschen Gesellschaft für Allergologie und klinische Immunologie (DGAI) und dem Ärzteverband Deutscher Allergologen (ÄDA) zu einer nachhaltigen Vertretung unserer Position und immerhin zu Teilerfolgen geführt hat. Die Auseinandersetzung ist jedoch noch lange nicht beendet. 1998 wird das 40jährige Bestehen der UEMS in Brüssel feierlich begangen. Wir werden uns dort auch als deutsche Vertreter einfinden und müssen bis dahin unsere „Lobby“ in Brüssel ganz erheblich verstärken.

Im niedergelassenen Bereich stellen sich immer mehr Kollegen die schwierige Frage, ob sie überhaupt noch sachgerechte Allergiediagnostik und -therapie mit den ständig neuen und zunehmend schwindenden Punktewerten betreiben können. Allenthalben stößt man auf Wut und Resignation gleichermaßen. Die deutschen Fachgesellschaften haben hierzu ihre Positionspapiere und Schriftsätze wiederholt und umfangreich abgegeben. Die Mehrheitsverhältnisse sprechen aber gegen die Spezialisten.

Unsere wirklichen Verbündeten sind die Patienten. Jeder Allergologe sollte jeden Fall, den er seiner Meinung nach nicht ausreichend oder optimal betreuen kann, melden. Überzeugende Einzelkasuistiken haben oft eine größere Wirkung als trockene Positionspapiere.

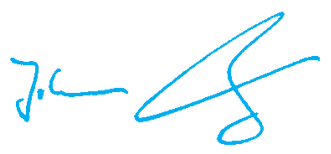

Prof. Dr. Dr. J. Ring
Völlig deletär wäre es, wenn sich niedergelassene Kollegen und Universitätskliniken gegeneinander ausspielen ließen. Die Interdisziplinarität der Allergologie verlangt darüber hinaus ein großes $\mathrm{Ma}$ an Toleranz zwischen den beteiligten Disziplinen. Deshalb sind Versuche, einzelne Fachdisziplinen gegen andere aufzuwiegeln (Pneumologen gegen Hals-Nasen-Ohren-Ärzte, Dermatologen gegen Pädiater etc.) in der Wurzel abzulehnen. Wenn wir uns auseinander dividieren lassen, ist die fachgerechte Allergologie in Deutschland ernsthaft gefährdet.

Es geht hier um die Patienten. Dies Argument müssen wir wie der alte $\mathrm{Ca}$ to in jede Debatte schleudern: „Ceterum censeo" zur Darstellung der Mißstände, dabei aber gleichzeitig kein trostloses Jammern.

Zum Ende des Halbjahres, das im Zeichen schwerer Auseinandersetzungen steht, dürfen wir auf keinen Fall resignieren, schon gar nicht mit dem Gedanken spielen „dann mache ich eben meine Praxis oder Klinik zu und tue etwas anderes Sinnvolles". Solche Gedanken sind der Anfang vom Ende. So würden sich die deutschen Allergologen selbst überflüssig machen. Das Heimtückische und Verwundbare an unserer Disziplin ist ja, daß mangelnde Sachkenntnis und damit verbundene Fehler in der Diagnostik, Behandlung und Prävention oft erst nach vielen Jahren für den Patienten bemerkbar werden.

Zum Ende des ersten Halbjahres 1997 wünschen die Herausgeber unseren Lesern eine erholsame Zeit mit Muße zur Besinnung und kreativen Gedanken!

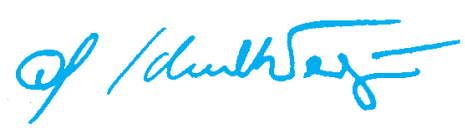

Prof. Dr. G. Schultze-Werninghaus 